\title{
In-Situ Environmental TEM Study of Solid-Gas Interfacial Process in Energy Materials
}

\author{
Lianfeng Zou and Chongmin Wang \\ Pacific Northwest National Laboratory, Richland, Washington, United States
}

Interfacial reactions between a base material and environments are ubiquitous and can deteriorate the performance of a rich variety of materials, including metals, semiconductors, and batteries. Under controlled conditions, however, some of the undesired reactions may become beneficial by assisting the surface or interface passivation, a process that leads to the formation of a stable shielding layer, as wellexemplified by the metal oxides for stainless steel and solid electrolyte interphase (SEI) layers in rechargeable. It is commonly observed that the state-of-the-art cathodes for rechargeable batteries, e.g., the nickel-rich and lithium-rich transition metal oxides, are susceptible to, during the stage of fabrication and storage, environmental degradations upon air exposure, a serious issue known as "air instability". Often, electrode instability stems from the cathode-air interfacial reactions by which the resultant products cause many practical issues such as cell degassing, slurry alkalization, electrolyte consumption, and irreversible electrode phase transition

In this work, we use atomic level environmental transmission electron microscopy to in-situ probe the dynamics of solid-gas interfacial reaction, including alloy oxidation, metal surface passivation, catalyst particle stability, functioning of metal-air battery, and cathode-air interfacial reactions. We intend to establish the fundamental principles for governing the solid-gas interfacial reaction. As a typical example, we choose the widely commercialized lithium-nickel-magnesium-cobalt based oxides (NMC), including $\mathrm{LiNi}_{1 / 3} \mathrm{Mn}_{1 / 3} \mathrm{Co}_{1 / 3} \mathrm{O}_{2}$ (NMC333), LiNi0.6 $\mathrm{Mn}_{0.2} \mathrm{Co}_{0.2} \mathrm{O}_{2}$ (NMC622), and LiNio.8 $\mathrm{Mn}_{0.1} \mathrm{Co}_{0.1} \mathrm{O}_{2}$ (NMC811), as a model system for systematic comparison study of their air-instability as a function of Ni content. By monitoring the interfacial reactions between the cathode and each isolated air component in real-time, we reveal that the cathode-air interfacial reaction is controlled by the $\mathrm{Li}^{+}$-water interactions driven delithiation, rather than chemical reaction. Characteristically, associated with the retarded $\mathrm{Li}$ ions diffusion in the reaction products/surface reconstruction layers, the reaction layer shows a critical thickness beyond which delithiation is arrested, thereby unlocking the potential of self-passivating for the cathode-air interfacial reactions[1].

The electrodes consisting of secondary particles were transferred to the FEI Helios Dual Beam system with an operation voltage ranges from 2 to $30 \mathrm{kV}$ was employed to do the lift out. Several random secondary particles of the electrode were targeted and the lift out processes were started by coating the targeted particles with Pt layers of $\sim 2 \mathrm{~mm}$ thickness. After that, a $\sim 3 \mathrm{~mm}$ cubic was extracted out of the secondary particle using focused ion beam (FIB), followed by attaching on the Cu TEM grid. The subsequent thinning processes was performed step by step using a voltage of $30 \mathrm{kV}$ until reach electron transparent thickness, the final polishing processes were carried out at a lower voltage of $5 \mathrm{kV}$ and $2 \mathrm{kV}$.

The as-prepared samples are then transferred to ETEM for the gas experiments. Before each gas is introduced for reactions and in situ observations, the TEM column was pumped to $10^{-8}$ Torr before the experiments and then a single gas was introduced into the column each time for the gas reactions. The beam current used for imaging is $\sim 2 \mathrm{nA}$. The STEM images were performed on the aberration corrected JEOL JEM-ARM200CF, at the operation voltage $200 \mathrm{kV}$. The electrons range from $90 \mathrm{mrad}$ to $370 \mathrm{mrad}$, and $10 \mathrm{mrad}$ to $23 \mathrm{mrad}$ were collected for STEM-HAADF, and STEM-ABF imaging, respectively. 
Passivation of electrodes has emerged as an effective means to tailor the rechargeable Li ion batteries for desired properties. Although the concept of electrochemical passivation is well-established with the discovery of electrode electrolyte interphase layers, the analogous chemical passivation in the atmospheric environments is, however, shrouded in mystery. Using atomic-resolution, time-resolved environmental transmission electron microscopy, we revealed that the chemical delithiation of air-instable cathode results in the formation of stable passivation phase, featuring a cathode air interface layer. The $\mathrm{Li}^{+} \mathrm{de}^{-}$ intercalation, driven by interaction with trace water, is self-limited by the ions transport through hydroxide films, dictating the formation of passivation films with a self-limiting thickness. Remarkably, the ultrathin passivation layers repair the local breakdowns in the self-healing manner. A comprehensive understanding of chemical passivation may assist the technological engineering of electrodes for long shelf life[2].

References

[1] Lianfeng Zou, Yang He, Zhenyu Liu, Haiping Jia, Jian Zhu, Jianming Zheng, Guofeng Wang, Xiaolin Li, Jie Xiao, Jun Liu, Ji-Guang Zhang, Guoying Chen and Chongmin Wang, "Unlocking the passivation nature of the cathode-air interfacial reactions in lithium ion batteries", Nature Communications, 2020, 11, 3204.

[2] The work was conducted in the William R. Wiley Environmental Molecular Sciences Laboratory (EMSL), a national scientific user facility sponsored by DOE's Office of Biological and Environmental Research and located at PNNL. 